\title{
Neutrinoless Double- $\beta$ Decay with Nonstandard Majoron Emission
}

\author{
Ricardo Cepedello, ${ }^{1,2, *}$ Frank F. Deppisch, ${ }^{2,3, \dagger}$ Lorena González, ${ }^{4,2, \$}$ Chandan Hati, ${ }^{5, \S}$ and Martin Hirsch ${ }^{1, \|}$ \\ ${ }^{1}$ AHEP Group, Instituto de Física Corpuscular-CSIC/Universitat de València Edificio de Institutos de Paterna, \\ Apartado 22085, E-46071 València, Spain \\ ${ }^{2}$ Department of Physics and Astronomy, University College London, London WCIE 6BT, United Kingdom \\ ${ }^{3}$ Institut für Hochenergiephysik, Österreichische Akademie der Wissenschaften, Nikolsdorfer Gasse 18, 1050 Wien, Austria \\ ${ }^{4}$ Department of Physics, Universidad Técnica Federico Santa María, Avenida España 1680, Valparaíso, Chile \\ ${ }^{5}$ Laboratoire de Physique de Clermont, CNRS/IN2P3 UMR 6533, Campus des Cézeaux, \\ 4 Avenue Blaise Pascal, F-63178 Aubière Cedex, France
}

(Received 13 November 2018; revised manuscript received 1 February 2019; published 7 May 2019)

\begin{abstract}
We present a novel mode of neutrinoless double- $\beta$ decay with emission of a light Majoron-like scalar particle $\phi$. We assume it couples via an effective seven-dimensional operator with a $(V+A)$ lepton current and $(V \pm A)$ quark currents leading to a long-range contribution that is unsuppressed by the light neutrino mass. We calculate the total double- $\beta$ decay rate and determine the fully differential shape for this mode. We find that future double- $\beta$ decay searches are sensitive to scales of the order $\Lambda_{\mathrm{NP}} \approx 1 \mathrm{TeV}$ for the effective operator and a light scalar $m_{\phi}<0.2 \mathrm{MeV}$, based on ordinary double- $\beta$ decay Majoron searches. The angular and energy distributions can deviate considerably from that of two-neutrino double- $\beta$ decay, which is the main background. We point out possible ultraviolet completions where such an effective operator can emerge.
\end{abstract}

DOI: 10.1103/PhysRevLett.122.181801

Introduction.-Double- $\beta$ decay processes are sensitive probes of physics beyond the standard model (SM). The SM process of two-neutrino double- $\beta(2 \nu \beta \beta)$ decay is the rarest process ever observed with half-lives of order $T_{1 / 2}^{2 \nu \beta \beta} \sim 10^{21}$ yr. Neutrinoless double- $\beta(0 \nu \beta \beta)$ decay, with no observation of any missing energy, is clearly the most important mode beyond the SM as it probes the Majorana nature and mass $m_{\nu}$ of light neutrinos, with current experiments sensitive as $T_{1 / 2}^{0 \nu \beta \beta} \sim\left(0.1 \mathrm{eV} / m_{\nu}\right)^{2} \times 10^{26} \mathrm{yr}$. In general, it is a crucial test for any new physics scenario that violates lepton number by two units.

On the other hand, one or more exotic neutral particles may also be emitted, with a signature of anomalous missing energy beyond that expected in $2 \nu \beta \beta$ decay. A well studied set of theories involve the emission of a scalar particle, called Majoron J. The first such proposed Majoron was a Goldstone boson associated with the spontaneous breaking of lepton number symmetry [1,2], coupling to a neutrino $\nu$ as $g_{J} \nu \nu J$, cf. Fig. 1 (left). Current searches have a sensitivity of the order $T_{1 / 2}^{0 \nu \beta \beta J} \sim\left(10^{-5} / g_{J}\right)^{2} \times 10^{24} \mathrm{yr}$. The term Majoron has been used in a wider sense, implying

Published by the American Physical Society under the terms of the Creative Commons Attribution 4.0 International license. Further distribution of this work must maintain attribution to the author(s) and the published article's title, journal citation, and DOI. Funded by SCOAP ${ }^{3}$. just a charge-neutral scalar particle (Goldstone boson or not) or vector particle [3]. Originally considered to be massless, it may also be a light particle [4-6] that can potentially be a dark matter candidate [7-9]. Searches for extra particles in double- $\beta$ decay are crucial in understanding neutrinos. Most importantly, violation of lepton number by two units and thus the Majorana nature of neutrinos can only be firmly established in the case of $0 \nu \beta \beta$ decay.

Not all such emission modes have been discussed in the literature. Existing experimental searches so far focus on the emission of one or two Majorons originating from the intermediate neutrino exchanged in the process. The different Majoron scenarios have been classified into several categories, all of which assume SM $(V-A)$ charged currents with the electrons and quarks. In this Letter, we

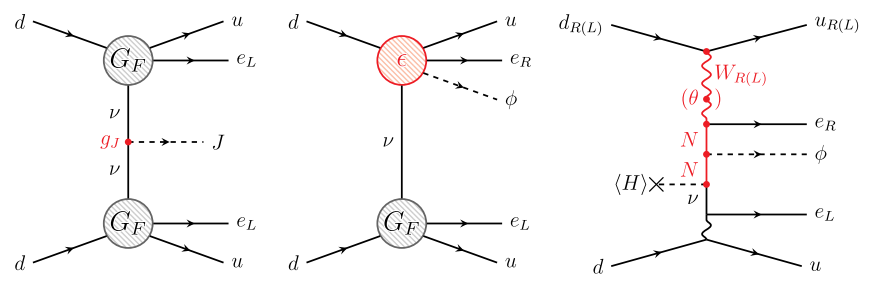

FIG. 1. Feynman diagrams for ordinary $0 \nu \beta \beta J$ Majoron decay (left), $0 \nu \beta \beta \phi$ decay triggered by an effective operator of the form $\Lambda_{\mathrm{NP}}^{-3}(\bar{u} \mathcal{O} d)(\bar{e} \mathcal{O} \nu) \phi$ (center), and possible ultraviolet completion of the latter in a left-right symmetric model (right). 
instead consider $0 \nu \beta \beta \phi$ decay with emission of a light neutral scalar $\phi$ from a single effective dimension-seven operator of the form $\Lambda_{\mathrm{NP}}^{-3}(\bar{u} \mathcal{O} d)(\bar{e} \mathcal{O} \nu) \phi$, cf. Fig. 1 (center), with the fermion currents having a different chiral structure from that in the SM. In the following, we will refer to the light scalar as "Majoron," independent of its origin. We determine the sensitivity to $\Lambda_{\mathrm{NP}}$ and analyze the effect on the energy and angular distributions in comparison with $2 \nu \beta \beta$ decay. We also comment on ultraviolet scenarios underlying the effective operator.

Effective long-range interactions.-We are interested in processes where right- and left-handed electrons are emitted along with a scalar $\phi$ considering as a first approach, only $(V+A)$ and $(V-A)$ currents. The effective Lagrangian can then be written as

$$
\begin{aligned}
\mathcal{L}_{0 \nu \beta \beta \phi}= & \frac{G_{F} \cos \theta_{C}}{\sqrt{2}}\left(j_{L}^{\mu} J_{L \mu}+\frac{\epsilon_{R L}^{\phi}}{m_{p}} j_{R}^{\mu} J_{L \mu} \phi+\frac{\epsilon_{R R}^{\phi}}{m_{p}} j_{R}^{\mu} J_{R \mu} \phi\right) \\
& + \text { H.c. },
\end{aligned}
$$

with the Fermi constant $G_{F}$, the Cabbibo angle $\theta_{C}$, and the leptonic and hadronic currents $j_{L, R}^{\mu}=\bar{e} \gamma^{\mu}\left(1 \mp \gamma_{5}\right) \nu$ and $J_{L, R}^{\mu}=\bar{u} \gamma^{\mu}\left(1 \mp \gamma_{5}\right) d$, respectively. Here, $\nu$ is a four-spinor field of the light electron neutrino, either defined by $\nu=$ $\nu_{L}+\nu_{L}^{c}$ (i.e., a Majorana spinor constructed from the SM active left-handed neutrino $\nu_{L}$ ) or $\nu=\nu_{L}+\nu_{R}$ (a Dirac spinor constructed from the SM $\nu_{L}$ and a new SM-sterile right-handed neutrino $\nu_{R}$ ). Whether the light neutrinos are of Majorana or Dirac type and whether total lepton number is broken or conserved is of crucial importance for an underlying model (determined by the chosen lepton numbers for $\nu_{R}$ and $\phi$ ) but as far as the effective interactions in Eq. (1) are concerned, this does not play a role in our calculations. The proton mass $m_{p}$ is introduced in the exotic interactions as normalization to make the effective coupling constants $\epsilon_{R L}^{\phi}$ and $\epsilon_{R R}^{\phi}$ dimensionless, in analogy to the effective operator treatment of $0 \nu \beta \beta$ decay [10,11]. In Eq. (1), we omit exotic operators with left-handed lepton currents; as in the standard long-range case, such contributions will be additionally suppressed by the small neutrino masses [11]. We instead focus on the process depicted in Fig. 1 (center), where the $\mathrm{SM}(V-A)$ Fermi interaction, the first term in Eq. (1), meets one of the exotic operators. In this case, the momentum part in the numerator of the neutrino propagator contributes, rather than the mass. In Eq. (1) we consider the first generation electron and neutrino only. Generalizing to three flavors amounts to promoting the $\epsilon_{R X}^{\phi}$ couplings to $3 \times 3$ matrices in generation space, $\left(\epsilon_{R X}^{\phi}\right)_{\alpha i}(\alpha=e, \mu, \tau$, $\left.i=\nu_{1}, \nu_{2}, \nu_{3}\right)$. The final decay rate will then be proportional to $\left|\epsilon_{R X}^{\phi}\right|^{2} \rightarrow\left|\sum_{i}\left(\epsilon_{R X}^{\phi}\right)_{e i} U_{e i}\right|^{2}$, where $U$ is the SM lepton mixing matrix.

Decay rate and distributions. - We base our calculation of the $0 \nu \beta \beta \phi$ decay rate and kinematic distributions on Doi et al. [12]. The details of the calculation are given in the Supplemental Material A [13]; here we outline the main features. Summing over all intermediate nuclear states $N$, the amplitude of $0_{I}^{+} \rightarrow 0_{F}^{+} 0 \nu \beta \beta \phi$ decay can be written as

$$
\begin{aligned}
\mathcal{M}= & \epsilon_{R X}^{\phi} \frac{\left(G_{F} \cos \theta_{C}\right)^{2}}{\sqrt{2} m_{p}} \sum_{N} \int d^{3} x d^{3} y \int \frac{d^{3} q}{2 \pi^{2} \omega} \phi(\mathbf{y}) e^{i \mathbf{q}(\mathbf{x}-\mathbf{y})} \\
& \times\left[\left(\frac{J_{L X}^{\rho \sigma}(\mathbf{x}, \mathbf{y}) u_{\rho \sigma}^{L}\left(E_{1} \mathbf{x}, E_{2} \mathbf{y}\right)}{\omega+\mu_{N}-\frac{1}{2}\left(E_{1}-E_{2}-E_{\phi}\right)}-\frac{J_{X L}^{\rho \sigma}(\mathbf{x}, \mathbf{y}) u_{\rho \sigma}^{R}\left(E_{1} \mathbf{x}, E_{2} \mathbf{y}\right)}{\omega+\mu_{N}-\frac{1}{2}\left(E_{1}-E_{2}+E_{\phi}\right)}\right)-\left(E_{1} \leftrightarrow E_{2}\right)\right] .
\end{aligned}
$$

Here, $X=L, R$ correspond to $\epsilon_{R L}^{\phi}, \epsilon_{R R}^{\phi}, \mu_{N}=E_{N}-E_{I}+$ $Q_{\beta \beta} / 2+m_{e}$ with $E_{I}$ and $E_{N}$ the energies of the initial and intermediate nucleus, respectively. The energies of the two outgoing electrons and the Majoron are $E_{1,2}$ and $E_{\phi}$, respectively, and the available kinetic energy release $Q_{\beta \beta}$. The nucleon and lepton currents are defined as

$J_{Y X}^{\rho \sigma}(\mathbf{x}, \mathbf{y})=\left\langle F\left|J_{Y}^{\rho}(\mathbf{x})\right| N\right\rangle\left\langle N\left|J_{X}^{\sigma}(\mathbf{y})\right| I\right\rangle, \quad X, Y=L, R$,

$u_{\rho \sigma}^{L, R}\left(E_{1} \mathbf{x}, E_{2} \mathbf{y}\right)=\frac{1}{2} q^{\mu} \bar{e}\left(E_{1} \mathbf{x}\right) \gamma_{\rho} \gamma_{\mu} \gamma_{\sigma}\left(1 \mp \gamma_{5}\right) e^{c}\left(E_{2} \mathbf{y}\right)$.

We consider that the internal neutrino propagates between the interaction points $\mathbf{x}$ and $\mathbf{y}$ with momentum $q^{\mu}=(\omega, \mathbf{q})$. From Eqs. (2) and (4) one can see explicitly the required antisymmetry of the amplitude under the exchange of the two electrons.

In Eq. (2), the Majoron energy $E_{\phi}$ is added or subtracted depending on whether the electron labeled 1 or 2 is being emitted from the exotic operator. The Majoron makes a crucial difference, as $E_{\phi}$ goes together with $\left(E_{1}-E_{2}\right)$ and not with the term proportional to the intermediate nuclei energy $\mu_{N}$ as for an ordinary Majoron. A dependence on $E_{\phi}$ will thus appear through the matrix element in addition to that through the phase space. The differential decay rate for the $0^{+} \rightarrow 0^{+} 0 \nu \beta \beta \phi$ decay can then be written as [12]

$$
\begin{aligned}
d \Gamma= & \frac{\left(G_{F} \cos \theta_{C} g_{A}\right)^{4} m_{e}^{2}}{256 \pi^{7}\left(m_{p} R\right)^{2}}\left[a\left(E_{1}, E_{2}\right)\right. \\
& \left.+b\left(E_{1}, E_{2}\right) \cos \theta\right] p_{1} p_{2} E_{1} E_{2} E_{\phi} d E_{1} d E_{2} d \cos \theta,
\end{aligned}
$$


TABLE I. Current limits and expected future sensitivity on the effective couplings $\epsilon_{R L}^{\phi}$ and $\epsilon_{R R}^{\phi}$ of $0 \nu \beta \beta \phi$ decay for ${ }^{82} \mathrm{Se}$ and ${ }^{136} \mathrm{Xe}$. The limits are estimated based on the experimental half-life constraints for ordinary Majoron emission (spectral index $n=1$ ) as given. Nuclear matrix elements from Refs. [35-37] where used for this estimate.

\begin{tabular}{lccc}
\hline \hline Isotope & $T_{1 / 2}[\mathrm{yr}]$ & $\left|\epsilon_{R L}^{\phi}\right|$ & $\left|\epsilon_{R R}^{\phi}\right|$ \\
\hline${ }^{82} \mathrm{Se}$ & $3.7 \times 10^{22}[38]$ & $4.1 \times 10^{-4}$ & $4.6 \times 10^{-2}$ \\
${ }^{136} \mathrm{Xe}$ & $2.6 \times 10^{24}[39]$ & $1.1 \times 10^{-4}$ & $1.1 \times 10^{-2}$ \\
${ }^{82} \mathrm{Se}$ & $1.0 \times 10^{24}$ & $8.0 \times 10^{-5}$ & $8.8 \times 10^{-3}$ \\
${ }^{136} \mathrm{Xe}$ & $1.0 \times 10^{25}$ & $5.7 \times 10^{-5}$ & $5.8 \times 10^{-3}$ \\
\hline \hline
\end{tabular}

with the axial coupling $g_{A}$ of the nucleon and the radius $R$ of the nucleus. The magnitudes of the electron spatial momenta are denoted $p_{1,2}$ and $0 \leq \theta \leq \pi$ is the angle between the emitted electrons. In Eq. (5), the Majoron energy is determined as $E_{\phi}=Q_{\beta \beta}+2 m_{e}-E_{1}-E_{2}$ by energy conservation. Definitions for the coefficients $a\left(E_{1}, E_{2}\right), b\left(E_{1}, E_{2}\right)$ in the decay rate can be found in the Supplemental Material A [13], where we show in detail the derivation of the differential decay rate. Therein we use the nuclear matrix elements listed in Table I and Coulombcorrected relativistic electron wave functions.

From Eq. (5), the total decay rate and thus the half-life is calculated by performing the integration of $a\left(E_{1}, E_{2}\right)$ over all energies within the allowed phase space limits $E_{1}$, $E_{2} \geq 0$ and $E_{1}+E_{2} \leq Q_{\beta \beta}+2 m_{e}$. In addition, we determine and discuss several distributions below. We will show results for the $\epsilon_{R L}^{\phi}$ and $\epsilon_{R R}^{\phi}$ versions of the effective operators where we consider only one of these to be present at a time. We assume the exotic $\phi$ Majoron to be massless in our calculations and comment on massive $\phi$ in the discussion below. For our numerical evaluation we focus on two isotopes: (i) ${ }^{136} \mathrm{Xe}$, for which the KamLANDZen collaboration [39] currently provides the most stringent constraints; (ii) ${ }^{82} \mathrm{Se}$ used by NEMO-3 and the upcoming SuperNEMO experiments [38] that can measure the detailed electron topology.

For all experimental searches, the crucial distribution is with respect to the sum of the kinetic energies of the detected electrons. With the SM $2 \nu \beta \beta$ decay as irreducible background to any exotic signal, it is important to calculate it precisely. In Fig. 2 (left), we compare the normalized total electron kinetic energy distribution of $0 \nu \beta \beta \phi$ decay with that of $2 \nu \beta \beta$ decay and ordinary $0 \nu \beta \beta J$ Majoron decay (with spectral index $n=1$ ) for the isotope ${ }^{136} \mathrm{Xe}$. The distribution associated with $\epsilon_{R L}^{\phi}$ is very similar to ordinary $0 \nu \beta \beta J$ decay, while the introduction of a hadronic righthanded current in the $\epsilon_{R R}^{\phi}$ term changes considerably the shape of the distribution. In both cases, the spectral index still corresponds to $n=1$ with the characteristic onset near the kinematic endpoint. We emphasize that because of the different shape, a dedicated signal over background analysis is required to determine the experimental sensitivity on the effective parameters $\epsilon_{R L}^{\phi}$ and $\epsilon_{R R}^{\phi}$ precisely.

NEMO-3 and SuperNEMO are able to measure the individual electron energies. In right-handed current scenarios without emission of a Majoron, the single energy distribution exhibits a distinctive valley-type shape. This occurs as the dominant term is proportional to $\left(E_{1}-E_{2}\right)$ for the corresponding $\epsilon_{R R}$ term, as a result of the antisymmetry with respect to electron exchange. (For the $\epsilon_{R L}$ term with a left-handed hadronic current, $P$-wave and nuclear recoil contribute constructively, giving a dominant contribution proportional to $\left(E_{1}+E_{2}\right)$ [12].) In our case, depicted in Fig. 2 (right), part of the energy is being carried away by the Majoron, shifting the distribution towards lower electron energies and softening the characteristic valley-type
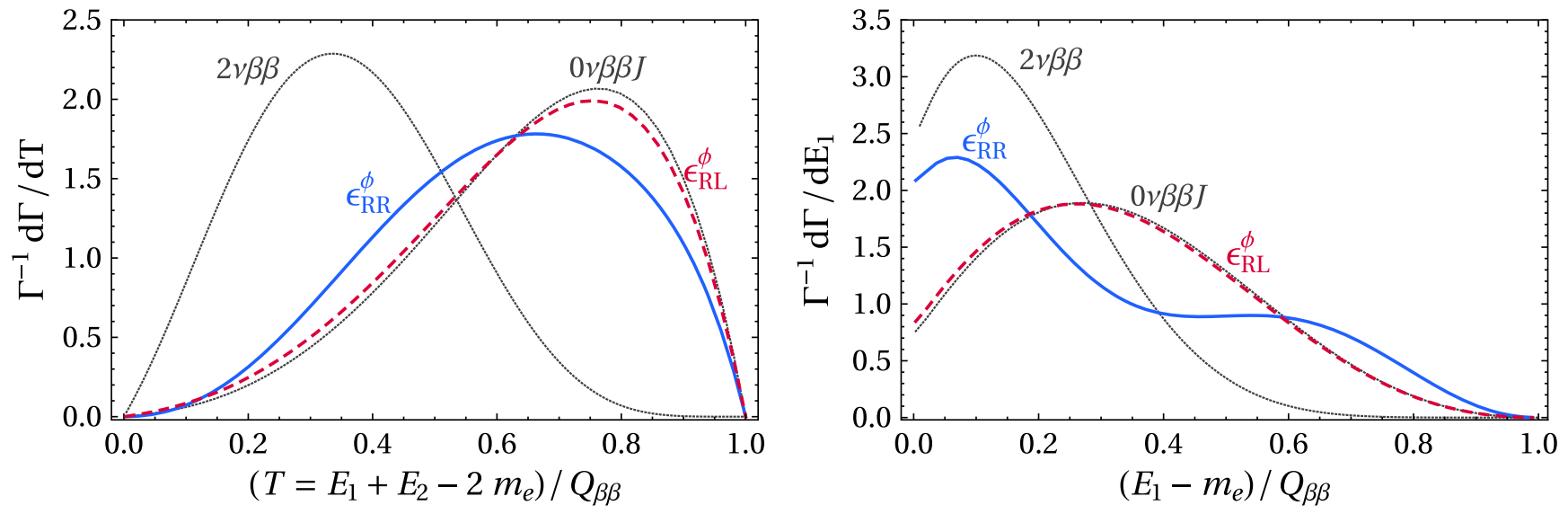

FIG. 2. Left: Normalized $0 \nu \beta \beta \phi$ decay distributions in the total kinetic energy of the electrons for ${ }^{136} \mathrm{Xe}$. Right: Normalized $0 \nu \beta \beta \phi$ decay distribution in the single electron kinetic energy distribution for ${ }^{82} \mathrm{Se}$. The blue solid and red dashed lines correspond to the $\epsilon_{R R}^{\phi}$ and $\epsilon_{R L}^{\phi}$ cases, respectively. The corresponding distributions for the SM $2 \nu \beta \beta$ decay and ordinary $0 \nu \beta \beta J$ Majoron decay (spectral index $n=1$ ) are given for comparison. 
distribution for $\epsilon_{R R}^{\phi}$. The distribution does not vanish for $E_{1}-m_{e}=\frac{1}{2} Q_{\beta \beta}$ (as in the ordinary right-handed current case), but is still significantly different from that of ordinary Majoron emission. The distribution with respect to both electron energies is depicted in Fig. 1 (top panel) in the Supplemental Material A [13]. It exhibits an even more pronounced difference between the $\epsilon_{R R}^{\phi}$ mode and $2 \nu \beta \beta$. This may be used experimentally to improve the sensitivity through kinematic selection criteria, counteracting the effect of the less peaked total energy distribution, cf. Fig. 2 (left).

One can use also angular correlations to distinguish between left-handed and right-handed currents [40,41], see Fig. 1 (bottom panel) in the Supplemental Material A [13]. Integrating over the electron energies one obtains the average angular distribution which takes the simple form $(d \Gamma / d \cos \theta)=(\Gamma / 2)(1+k \cos \theta)$. The coefficient $k$ is $k_{R L}^{\phi}=+0.70$ (electrons are dominantly emitted collinearly) and $k_{R R}^{\phi}=-0.05$ (electrons are emitted nearly isotropically) in our $0 \nu \beta \beta \phi$ scenarios with $\epsilon_{R L}^{\phi}$ and $\epsilon_{R R}^{\phi}$, respectively, for ${ }^{82} \mathrm{Se}$. For comparison, the angular correlation factor for SM $2 \nu \beta \beta$ decay is $k^{2 \nu \beta \beta}=-0.66$ and $k^{J}=-0.80$ for ordinary Majoron emission; i.e., the electrons are dominantly emitted back to back.

Finally, we estimate the sensitivity of existing and planned future double- $\beta$ decay searches on the effective coupling strength $\epsilon_{R L}^{\phi}$ and $\epsilon_{R R}^{\phi}$ of $0 \nu \beta \beta \phi$ decay. We would like to emphasize again that due to the different total electron energy distribution, a dedicated signal over background analysis is required to determine the constraints precisely. Experiments such as NEMO-3 and SuperNEMO can also improve their sensitivity due to the nonstandard decay topology, especially for $\epsilon_{R R}^{\phi}$. As detailed in the Supplemental Material A [13], a requirement that any one electron has a kinetic energy of $E_{i}-m_{e}>Q_{\beta \beta} / 2$ can for example reduce the $2 \nu \beta \beta$ background by an order of magnitude. Here, we simply estimate the sensitivity by comparing our predictions for the $0 \nu \beta \beta \phi$ decay half-life $T_{1 / 2}=\ln 2 / \Gamma$ with the experimental constraints on ordinary $(n=1)$ Majoron emission. We use the most stringent limits for ${ }^{82} \mathrm{Se}$ by NEMO-3 [38] and for ${ }^{136} \mathrm{Xe}$ by KamLAND-Zen [39]. For future prospects, we estimate that experimental Majoron search sensitivities may reach $T_{1 / 2}^{\mathrm{Se}} \approx 10^{24} \mathrm{yr}$ (e.g., with the help of angular and energy selection cuts at SuperNEMO) and $T_{1 / 2}^{\mathrm{Xe}} \approx 10^{25} \mathrm{yr}$. (The corresponding $0 \nu \beta \beta$ decay sensitivities of the planned SuperNEMO [42] and nEXO experiments [43] may improve by $\mathcal{O}(100)$, but this requires an experimental approach that is essentially background-free. This is not possible for Majoron emission with a continuous total electron energy spectrum.) The corresponding limits on $\epsilon_{R L}^{\phi}$ and $\epsilon_{R R}^{\phi}$ are shown in Table I, where only one effective operator is assumed to present at a time.
Discussion.-Searches for Majorons or Majoron-like particles are a staple in double- $\beta$ decay experiments. So far, they only cover the case where the neutrino involved couples via the SM $(V-A)$ charged current interaction. This is clearly a well-motivated minimal choice but it is worthwhile to explore other scenarios. In this Letter, we have discussed one such alternative where a Majoron-like particle $\phi$ is emitted from effective operators with $(V+A)$ leptonic currents, cf. Fig 1 (center). The future sensitivities on the effective couplings $\epsilon_{R L}^{\phi}$ and $\epsilon_{R R}^{\phi}$ shown in Table I may be translated into effective operator scales $\Lambda_{\mathrm{NP}} \approx$ $1.3 \mathrm{TeV}$ and $270 \mathrm{GeV}$, respectively, using $1 / \Lambda_{\mathrm{NP}}^{3}=$ $\epsilon_{R X}^{\phi} G_{F} \cos \theta_{C} /\left(\sqrt{2} m_{p}\right)$. As noted before, we assume a massless $\phi$ in deriving these limits; they remain essentially unchanged for masses small compared to $Q_{\beta \beta}, m_{\phi} \lesssim$ $0.2 \mathrm{MeV}$ and are of the same order for $m_{\phi} \lesssim 1 \mathrm{MeV}$, but will deteriorate as $m_{\phi} \rightarrow Q_{\beta \beta}$ (for a recent analysis in ordinary Majoron emission, see Ref. [9]). Constraints on our operators may also be set from other processes, such as exotic decay modes of the pion, $\pi^{-} \rightarrow e^{-} \bar{\nu}_{e} \phi$. As we consider only $V+A$ currents, helicity suppression will still apply and the limits are expected to be correspondingly weak, we roughly estimate $\Lambda_{\mathrm{NP}} \gtrsim 15 \mathrm{GeV}$.

An ultraviolet scenario generating the effective operators in Eq. (1) is suggested in left-right symmetric models [44-47] where the SM $W$ and $\nu$ are replaced by their right-handed counterparts $W_{R}$ and $N$. The heavy neutrino $N$ then mixes with $\nu$ via a Yukawa coupling $y_{\nu}$ once the SM Higgs boson acquires its vacuum expectation value $\langle H\rangle=174 \mathrm{GeV}$. A massless or light scalar $\phi$ is not part of the minimal left-right symmetric model which thus needs to be modified, e.g., by keeping the $U(1)_{B-L}$ symmetry global or by extending its scalar sector. Charging $\phi$ under lepton number allows coupling to $N$ with a strength $y_{N}$. The corresponding diagram is shown in Fig. 1 (right). We can then identify

$$
\frac{G_{F} \cos \theta_{C}}{\sqrt{2} m_{p}} \epsilon_{R R}^{\phi}=\frac{g_{R}^{2} y_{N} y_{\nu}\langle H\rangle \cos \theta_{C}^{R}}{8 m_{W_{R}}^{2} m_{N}^{2}},
$$

leading to the estimate

$\frac{T_{1 / 2}^{\mathrm{Xe}}}{10^{25} \mathrm{yr}} \approx\left(\frac{3.5 \times 10^{-4}}{g_{R}^{2} y_{N} y_{\nu} \cos \theta_{C}^{R}}\right)^{2}\left(\frac{m_{W_{R}}}{4 \mathrm{TeV}}\right)^{4}\left(\frac{m_{N}}{100 \mathrm{MeV}}\right)^{4}$,

where $g_{R}$ is the gauge coupling constant and $\theta_{C}^{R}$ the equivalent of the Cabibbo angle, both associated with the $S U(2)_{R}$ of the left-right symmetric model. Alternatively, it is also possible to trigger the $\epsilon_{R L}^{\phi}$ mode through the $W_{R^{-}} W$ mixing $\theta$. Its value is generically expected to be $\theta=\kappa g_{R} m_{W}^{2} /\left(g_{L} m_{W_{R}}^{2}\right)$ where $\kappa=\mathcal{O}(1)$. In this case one has 


$$
\frac{G_{F} \cos \theta_{C}}{\sqrt{2} m_{p}} \epsilon_{R L}^{\phi}=\frac{g_{R} g_{L} \theta y_{N} y_{\nu}\langle H\rangle \cos \theta_{C}}{8 m_{W}^{2} m_{N}^{2}},
$$

resulting in the estimate

$$
\frac{T_{1 / 2}^{\mathrm{Xe}}}{10^{25} \mathrm{yr}} \approx\left(\frac{1.4 \times 10^{-4}}{g_{R}^{2} \kappa y_{N} y_{\nu}}\right)^{2}\left(\frac{m_{W_{R}}}{25 \mathrm{TeV}}\right)^{4}\left(\frac{m_{N}}{100 \mathrm{MeV}}\right)^{4} .
$$

This is more stringent due to the better sensitivity on $\epsilon_{R L}^{\phi}$ in Table I. Choosing the right-handed neutrino mass $m_{N}$ to be as low as $100 \mathrm{MeV}$ is strictly speaking not allowed in the effective operator treatment which requires $m_{N} \gg p_{F} \approx 100 \mathrm{MeV}$, but it may be more natural in a scenario where the mass of $N$ is generated through the vacuum expectation value of $\phi, m_{N}=y_{N}\langle\phi\rangle$. In fact, choosing $m_{N}$ to be smaller and abandoning the effective operator treatment may be more natural; the qualitative arguments should hold as above though a dedicated calculation of $0 \nu \beta \beta \phi$ would be required. In addition, the contribution to $0 \nu \beta \beta \phi$ via a heavy neutrino is expected to peak at $m_{N} \approx p_{F}$ with the above estimates applying to a good approximation [48]. (In addition to the operators discussed here, the left-right symmetric scenario will also induce a standard Majoron interaction $\phi \nu \nu$ (leading to standard Majoron emission with spectral index $n=1$ ) after electroweak symmetry breaking from an operator of the form $\phi H H \nu \nu$. It is suppressed relative to our contributions by an additional power of $y_{\nu}$ but does not suffer from suppression by the heavy $W_{R}$ mass or the small $W_{R}-W$ mixing.) We can here only give a sketch of what such a model scenario may look like; for a more detailed discussion, we refer the reader to our Supplemental Material B.1 [13] where we describe a left-right symmetric model incorporating a Dirac seesaw mechanism to generate the light neutrino masses. Here, ordinary $0 \nu \beta \beta$ decay is not allowed and only our $0 \nu \beta \beta \phi$ mode would occur.

Other ultraviolet completions do exist; to lowest dimension, the effective operator $\epsilon_{R R}^{\phi}$ in Eq. (1) can be matched to the SM invariant operator $L e_{R} \bar{d}_{R} u_{R} H \phi \quad\left(=\mathcal{O}_{8} \phi\right.$ in the counting of lepton number violating operators in Ref. [49]). All tree level completions of the operator $\mathcal{O}_{8}$ were derived in Ref. [50] which can be easily adapted to include the SM singlet $\phi$. These for example include heavy leptoquarks as well as heavy scalars and fermions as present in $R$-parity violating supersymmetry, cf. Supplemental Material B.2 [13] for more details. The interactions in Eq. (1) could also be extended in several directions. Most straightforwardly, one can generalize Eq. (1) by including scalar and tensor fermion currents to incorporate all possible Lorentz-invariant combinations. The Majoron may also couple derivatively, if originating as a Goldstone boson; this would increase the number of possible Lorentz-invariant combinations. Alternatively, if the exotic particle is a vector boson $a^{\mu}$ [3], such as a dark photon, the fermion currents can couple to it via the vector field itself as well as its field strength tensor $f^{\mu \nu}$. An even number of exotic neutral fermions $\chi$ may also be emitted but this would quickly increase the dimension of the corresponding effective operator. Instead, they may also originate from the internal neutrino via a dimension-six operator of the form $\Lambda_{\mathrm{NP}}^{-2} \nu \nu \chi \chi$ [51]. Exploring such alternatives to the well-studied neutrinoless double- $\beta$ decay is imperative in order to be able to draw reliable conclusions on the nature of neutrino mass generation.

The work of R.C. and M.H. was supported by the Spanish Grants No. FPA2017-85216-P (MICINN), No. SEV-2014-23510398 (AEI/FEDER, UE), No. PROMETEO/2018/165 (Generalitat Valenciana), Red Consolider MultiDark No. FPA2017-90566-REDC (MICINN), No. FPU15/03158, and No. EST17/00328. The work of F.F.D. was supported by the UK STFC and a Royal Society Exchange Grant. The work of L. G. was supported by Conicyt Chile under Grant No. 21160645 and DGIIP of UTFSM. C. H. acknowledges support within the framework of the European Union's Horizon 2020 research and innovation programme under the Marie Sklodowska-Curie Grant Agreements No. 690575 and No. 674896. R. C. and L. G. would like to thank the UCL Department of Physics for its hospitality.

*ricepe@ific.uv.es

†f.deppisch@ucl.ac.uk

†orena.gonzalez@alumnos.usm.cl

§chandan.hati@clermont.in2p3.fr

"mahirsch@ific.uv.es

[1] Y. Chikashige, R. N. Mohapatra, and R. D. Peccei, Phys. Lett. 98B, 265 (1981).

[2] G. B. Gelmini and M. Roncadelli, Phys. Lett. 99B, 411 (1981).

[3] C. D. Carone, Phys. Lett. B 308, 85 (1993).

[4] P. Bamert, C. P. Burgess, and R. N. Mohapatra, Nucl. Phys. B449, 25 (1995).

[5] M. Hirsch, H. V. Klapdor-Kleingrothaus, S. G. Kovalenko, and H. Päs, Phys. Lett. B 372, 8 (1996),

[6] K. Blum, Y. Nir, and M. Shavit, Phys. Lett. B 785, 354 (2018).

[7] V. Berezinsky and J. W. F. Valle, Phys. Lett. B 318, 360 (1993).

[8] C. Garcia-Cely and J. Heeck, J. High Energy Phys. 05 (2017) 102.

[9] T. Brune and H. Päs, arXiv:1808.08158.

[10] H. Päs, M. Hirsch, H. V. Klapdor-Kleingrothaus, and S. G. Kovalenko, Phys. Lett. B 453, 194 (1999).

[11] F. F. Deppisch, M. Hirsch, and H. Päs, J. Phys. G 39, 124007 (2012).

[12] M. Doi, T. Kotani, and E. Takasugi, Prog. Theor. Phys. Suppl. 83, 1 (1985).

[13] See Supplemental Material at http://link.aps.org/ supplemental/10.1103/PhysRevLett.122.181801 for details about the calculation and for some examples of ultraviolet complete scenarios, which includes Refs. [14-34]. 
[14] G. Senjanovic, Nucl. Phys. B153, 334 (1979).

[15] G. R. Dvali, G. Lazarides, and Q. Shafi, Phys. Lett. B 424, 259 (1998).

[16] K. S. Babu, J. C. Pati, and F. Wilczek, Nucl. Phys. B566, 33 (2000).

[17] S. M. Barr, Phys. Rev. Lett. 92, 101601 (2004).

[18] C. H. Albright and S. M. Barr, Phys. Rev. D 69, 073010 (2004).

[19] C. H. Albright and S. M. Barr, Phys. Rev. D 70, 033013 (2004).

[20] A. Davidson and K. C. Wali, Phys. Rev. Lett. 59, 393 (1987).

[21] S. Rajpoot, Phys. Rev. D 36, 1479 (1987).

[22] D. Chang and R. N. Mohapatra, Phys. Rev. Lett. 58, 1600 (1987).

[23] B. S. Balakrishna, Phys. Rev. Lett. 60, 1602 (1988).

[24] K. S. Babu and R. N. Mohapatra, Phys. Rev. Lett. 62, 1079 (1989).

[25] K. S. Babu and R. N. Mohapatra, Phys. Rev. D 41, 1286 (1990).

[26] B. Brahmachari, E. Ma, and U. Sarkar, Phys. Rev. Lett. 91, 011801 (2003).

[27] H. Murayama and A. Pierce, Phys. Rev. Lett. 89, 271601 (2002).

[28] B. Thomas and M. Toharia, Phys. Rev. D 73, 063512 (2006).

[29] B. Thomas and M. Toharia, Phys. Rev. D 75, 013013 (2007).

[30] S. Abel and V. Page, J. High Energy Phys. 05 (2006) 024.

[31] D. G. Cerdeno, A. Dedes, and T. E. J. Underwood, J. High Energy Phys. 09 (2006) 067.

[32] P.-H. Gu and H.-J. He, J. Cosmol. Astropart. Phys. 12 (2006) 010.

[33] P.-H. Gu, H.-J. He, and U. Sarkar, Phys. Lett. B 659, 634 (2008).
[34] P.-H. Gu, H.-J. He, and U. Sarkar, J. Cosmol. Astropart. Phys. 11 (2007) 016.

[35] M. Horoi and A. Neacsu, Phys. Rev. D 93, 113014 (2016).

[36] E. Caurier, F. Nowacki, A. Poves, and J. Retamosa, Phys. Rev. Lett. 77, 1954 (1996).

[37] E. Caurier, F. Nowacki, and A. Poves, Eur. Phys. J. A 36, 195 (2008).

[38] R. Arnold et al., Eur. Phys. J. C 78, 821 (2018).

[39] A. Gando et al. (KamLAND-Zen Collaboration), Phys. Rev. Lett. 117, 082503 (2016); 117, 109903(A) (2016).

[40] M. Doi, T. Kotani, H. Nishiura, and E. Takasugi, Prog. Theor. Phys. 70, 1353 (1983).

[41] R. Arnold et al. (SuperNEMO Collaboration), Eur. Phys. J. C 70, 927 (2010).

[42] C. Macolino (SuperNEMO Collaboration), Proc. Sci. EPS-HEP2017 (2017) 121

[43] G. Gratta, Neutrino 2018: Neutrinoless Double Beta Decay with EXO-200 and nEXO, 2018.

[44] J. C. Pati and A. Salam, Phys. Rev. D 10, 275 (1974); 11, 703(E) (1975).

[45] R. N. Mohapatra and J. C. Pati, Phys. Rev. D 11, 566 (1975).

[46] R. N. Mohapatra and J. C. Pati, Phys. Rev. D 11, 2558 (1975).

[47] G. Senjanovic and R. N. Mohapatra, Phys. Rev. D 12, 1502 (1975).

[48] F. Simkovic, G. Pantis, J. D. Vergados, and A. Faessler, Phys. Rev. C 60, 055502 (1999).

[49] K. S. Babu and C. N. Leung, Nucl. Phys. B619, 667 (2001).

[50] J. C. Helo, M. Hirsch, and T. Ota, J. High Energy Phys. 06 (2016) 006.

[51] W.-C. Huang and F. F. Deppisch, Phys. Rev. D 91, 093011 (2015). 